\title{
RESEARCH HIGHLIGHTS IN IAS
}

\section{MARKO KREFT}

Department of Biology, Biotechnical Faculty, University of Ljubljana, Večna pot 111, 1000 Ljubljana, Slovenia; Laboratory of Neuroendocrinology-Molecular Cell Physiology, Institute of Pathophysiology, Faculty of Medicine, University of Ljubljana, Zaloška 4, 1000 Ljubljana, Slovenia; Celica Biomedical, Tehnološki park 24, 1000 Ljubljana, Slovenia

e-mail: marko.kreft@bf.uni-lj.si

\begin{abstract}
We are reviewing and commenting highlights of the research published in Image Analysis and Stereology journal (IAS), volume 35, where 16 original research papers on image analysis, computer vision, modelling, and other approaches were published. We have reported on the precision of curve length estimation in the plane. Further, a focus was on a robust estimation technique for 3D point cloud registration. Next contribution in computer vision was on the accuracy of stereo matching algorithm based on illumination control. An attempt was also made to automatically diagnose prenatal cleft lip with representative key points and identify the type of defect in three-dimensional ultrasonography. Similarly, a new report is presenting estimation of torsion of digital curves in 3D images and next, the nuchal translucency by ultrasound is being analyzed. Also in ophthalmology, image analysis may help physicians to establish a correct diagnosis, which is supported by a new approach to measure tortuosity of retinal vessel. Another report of medical significance analyzed correlation of the shape parameters for characterization of images of corneal endothelium cells. Shape analysis is also an important topic in material science, e.g. in analyzing fine aggregates in concrete. As in concrete, in fiber reinforced composites image analysis may aid in improved quality, where the direction of fibers have decisive impact on properties. Automatic defect detection using a computer vision system improves productivity quality in industrial production, hence we report of a new Haar wavelet-based approach.
\end{abstract}

Keywords: computer vision, image analysis, process modelling, stereology

In the first issue of the volume 35 in 2016 we reported on the precision of curve length estimation in the plane. The study was based on the intersection counting with a square grid, called the BuffonSteinhaus estimator. This is relatively straightforward, design unbiased and efficient approach. The outstanding task was the prediction of its error variance from a single grid superimposition. Authors considered nine curvilinear features - that is flattened DNA molecule projections. Authors explain that the performance of their variance prediction formula is well suited for practical use (Gomez et al., 2016).

Further, a focus was on a robust estimation technique for 3D point cloud registration. Usually, the 3D vision applications require a complete or registered $3 \mathrm{D}$ image of the object. The automatic pairwise coarse alignment of partially overlapping 3D images is performed. We reported on a novel algorithm for the $3 \mathrm{D}$ registration task which is faster and more accurate than the usual RANSAC (Random Sample Consensus) algorithm (Pankaj and Nidamanuri, 2016). Next contribution in computer vision was on the accuracy of stereo matching algorithm based on illumination control. It is a new method of pixel based stereo matching algorithm. The standard algorithm for absolute difference is fast but lacs precision at low texture areas and is sensitive to radiometric distortions. The new suggested algorithm utilizes an illumination control which leads to enhancement of the image quality of absolute difference matching. The discontinuity errors are reduced, which leads to a significant enhancement on matching quality and accuracy of disparity maps (Hamzah et al., 2016).

In a next paper we described the new segmentation approach. Graph cut segmentation analyses images in a globally optimized fashion, however, finding a suitable parameter value that adjusts its data term and smoothness term may be a challenge. We report of an adaptive parameter selection. The extracted cell boundary dynamically adjusts the parameter value of the graph cut, countering its shrink bias. The approach was shown to be promising and may be used 
in medical diagnosis (Oyebode and Tapamo, 2016). An attempt was also made to automatically diagnose prenatal cleft lip with representative key points and identify the type of defect in three-dimensional ultrasonography. Facial shapes and curvatures have been geometrically described. The descriptive accuracy of the descriptors of the typical key points enables automatic extractions of reference points, quantitative distances, labial profiles, and provides information about facial asymmetry. Cleft lip was correctly diagnosed and characterized in all tested cases. It seems promising that the algorithm will help practitioners in identifying and classifying cleft lips (Vezzetti et al., 2016).

In the second issue of the volume 35 we reported on the content based video retrieval based on Hadamard matrix and discrete wavelet transform (HDWT). Content based video retrieval (CBVR) systems are useful for a wide range of applications. In this issue we report a video retrieval using the visual information from the video. The local and global color, texture, and motion features of the video are extracted as features of key frames. The applicability of the proposed technique was superior compared to the other methods however the use of video query instead of frame query may further improve the proposed method (Mohamadzadeh and Farsi, 2016).

The new report presents estimation of torsion of digital curves in 3D images. Torsion has an exact definition in the continuous domain, whereas in the discrete domain torsion evaluation is less definite. The use of the discrete points of space curves is presented to determine the Fourier series coefficients which allow representation of continuous curve with Cesàro's mean. This approach has an advantage, that no a priori information about the shape of the curve parts is needed. The algorithm was tested with a synthetic data, as well as with the tomographic data of fiber structures and open foams (Blankenburg et al., 2016).

Field programmable gate array (FPGA) was used to extract road network in remote sensing, where morphological operator is necessary to extract the road network from satellite images. The use of hardware for processing is compared with the dedicated software and both approaches were referenced to the road map (Sujatha and Selvathi, 2016).

The nuchal translucency by ultrasound is a useful diagnostic procedure for screening for the Down's syndrome in the first trimester of pregnancy, where the expertise of the physician is crucial. In IAS journal we published a new methodology to support the physician in the automatic identification of mid-sagittal sections of the fetus in ultrasound videos. The method proposed is based on wavelet analysis and neural network classifiers to detect the jawbone and on radial symmetry analysis to detect the choroid plexus, which enables identification of the frames which represent appropriate mid-sagittal sections. The results were compared with physician decisions (Sciortino et al., 2016).

Also in ophthalmology, image analysis may help physicians to establish the correct diagnosis. We published a new approach to measure tortuosity of retinal vessel. This is important in retinopathies, which are one of major causes of blindness. The approach described uses the combination of difference image and $\mathrm{k}$-means clustering for the segmentation of retinal vessels. Stationary points in the vessel center-lines are used to model the detection of twists in the vessel segments. The proposed technique yields high mean sensitivity and mean accuracy rates at good specificity (Mapayi et al., 2016).

In the last issue of IAS volume 35 we first addressed an ensemble classification scheme, employing the mutual information, the cross-correlation and the clustering based on proximity of image features methods, for early stage assessment of melanomas on plain photography images. It is a two stage process; first it retrieves the most similar image from samples of verified benign moles and malignant melanoma cases. Second, it provides an automated estimation regarding the nature of the unknown image. The proposed scheme for diagnostic aid may benefit patients in decision making especially for ambiguous cases (Kostopoulos et al., 2016). Another report of medical significance analyzed correlation of the shape parameters for characterization of images of corneal endothelium cells. Typically, such examination is manual which is time consuming. The report compared different shape measures to be combined in order to improve descriptions (Nurzynska and Piorkowski, 2016). Shape analysis is also an important topic in material science, e.g. in analyzing fine aggregates in concrete. Shape characteristics of fine aggregates are compared with coarse aggregates with one outstanding shape parameter: bluntness. This image analysis method is shown to be appropriate for the shape characterization of fine aggregate in concrete (He et al., 2016).

As in concrete, in fiber reinforced composites image analysis may aid in improved quality, where the direction of fibers have decisive impact on properties. The report in the last issue of IAS shows how fiber 
direction distribution is related to quantities commonly used in engineering applications. Four algorithms for local orientation analysis are discussed: those based on the response of anisotropic Gaussian filters, moments and axes of inertia derived from directed distance transforms, the structure tensor, and the Hessian matrix (Wirjadi et al., 2016).

In material sciences, in biology and medicine modelling processes is increasingly important, one of possible model being random sets. We published a study of a measure of dissimilarity of stationary and isotropic random sets through a heuristic based on convex compact approximations, support functions and envelope tests (Gotovac et al., 2016).

Automatic defect detection using a computer vision system improves productivity quality in industrial production. We report of a new Haar wavelet-based approach, which explores space localization properties of the discrete Haar wavelet transform and generates statistically-based parameterized texture defect detection criteria. The approach was tested on industrial analyses samples of ceramic tiles, glass samples, and textile (Vaideliene and Valantinas, 2016).

\section{REFERENCES}

Blankenburg C, Daul C, Ohser J (2016). Estimating torsion of digital curves using $3 \mathrm{~d}$ image analysis. Image Anal Stereol 35:81-91.

Gomez A, Cruz M, Cruz-Orive L (2016). On the precision of curve length estimation in the plane. Image Anal Stereol 35:1-14.

Gotovac V, Helisova K, Ugrina I (2016). Assessing dissimilarity of random sets through convex compact approximations, support functions and envelope tests. Image Anal Stereol 35:181-93.

Hamzah R, Ibrahim H, Abu Hassan A (2016). Stereo matching algorithm based on illumination control to improve the accuracy. Image Anal Stereol 35:39-52.

He H, Courard L, Pirard E, Michel F (2016). Shape analysis of fine aggregates used for concrete. Image Anal Stereol 35:159-66.

Kostopoulos S, Glotsos D, Asvestas P, Konstandinou C, Xenogiannopoulos G, Sidiropoulos K, Nikolatou E, Perakis K, Mantzouratos S, Sakkis T, Sakellaropoulos G, Nikiforidis G, Cavouras D (2016). An ensemble template matching and content-based image retrieval scheme towards early stage detection of melanoma. Image Anal Stereol 35:137-48.

Mapayi T, Tapamo J, Viriri S, Adio A (2016). Automatic retinal vessel detection and tortuosity measurement. Image Anal Stereol 35:117-35.
Mohamadzadeh S, Farsi H (2016). Content based video retrieval based on hdwt and sparse representation. Image Anal Stereol 35:67-80.

Nurzynska K, Piorkowski A (2016). The correlation analysis of the shape parameters for endothelial image characterisation. Image Anal Stereol 35:149-58.

Oyebode K, Tapamo J (2016). Adaptive parameter selection for graph-cut based segmentation on cell images. Image Anal Stereol 35:29-37.

Pankaj D, Nidamanuri R (2016). A robust estimation technique for $3 \mathrm{~d}$ point cloud registration. Image Anal Stereol 35:15-28.

Sciortino G, Orlandi E, Valenti C, Tegolo D (2016). Wavelet analysis and neural network classifiers to detect mid-sagittal sections for nuchal translucency measurement. Image Anal Stereol 35:105-15.

Sujatha C, Selvathi D (2016). Fpga implementation of road network extraction using morphological operator. Image Anal Stereol 35:93-103.

Vaideliene G, Valantinas J (2016). The use of haar wavelets in detecting and localizing texture defects. Image Anal Stereol 35:195-201.

Vezzetti E, Speranza D, Marcolin F, Fracastoro G (2016). Diagnosing cleft lip pathology in 3d ultrasound: A landmarking-based approach. Image Anal Stereol 35:53-65.

Wirjadi O, Schladitz K, Easwaran P, Ohser J (2016). Estimating fibre direction distributions of reinforced composites from tomographic images. Image Anal Stereol 35:167-79. 